\title{
Dirichlet Series and Convolution Equations
}

\author{
By \\ Carlos A. Berenstein* and Daniele C. Struppa**
}

\section{$\S 0$. Introduction}

In this paper we wish to propose a totally new approach to the theory of Dirichlet series, with the hope that our method will be able to shed new light on some old problems in the area.

Our viewpoint is to consider Dirichlet series as a very particular case of solutions of homogeneous convolution equations, and to consider these objects in the framework of a general theory of Fourier analysis on non-algebraic varieties.

In view of the character of this paper (which has to be considered preliminary, even though a relatively large number of results is obtained), we would like to briefly describe the philosophy which underlies our approach. From a historical point of view, the theory of Fourier integrals has been devised to be able to represent an "arbitrary" function as a linear combination of exponentials. Thus, functions on $\boldsymbol{R}$ (or $\boldsymbol{R}^{n}$ ) which are sufficiently small at infinity, were given such representations with the use of exponentials with real frequencies. When larger growths at infinity had to be handled, it became necessary to allow the use of exponentials with complex frequencies and, as an immediate consequence, the Fourier integral representations became essentially non-unique, as every exponential $\exp (x \cdot z), z \in \boldsymbol{C}$, has many integral representations (via the Cauchy formula) as a limit of linear combinations of exponentials. In the sixties, these natural considerations led L. Ehrenpreis to consider Fourier integral representations of (for example) $C^{\infty}$ functions, with the specific purpose of finding out the existence of subsets $S$ of $\boldsymbol{C}$ (which Ehrenpreis called sufficient sets) such that integral representations could exist with frequencies in $S$. Ehrenpreis developed a quite powerful theory (the so called theory of $A U$-spaces [7]) with the idea of studying integral representations with exponentials whose frequencies were restricted to belong to some algebraic variety in $C$ (or $\boldsymbol{C}^{n}$ ). This study, in particular, provided a wealth of results on solutions of systems of linear constant coefficients partial differential equations (in which case $S$ is the

Communicated by M. Kashiwara, May 9, 1988.

* Department of Mathematics, University of Maryland, College Park, MD 20742, USA.

** Dipartimento di Matematica, Università della Calabria, 87036 Arcavacata di Rende (CS), Italy.

*** This research was supported in part by an NSF Grant. 
algebraic variety known as the characteristic variety of the system). This is essentially the content of Parts A and B of [7].

It turns out, however, that there are many interesting cases in which one cannot restrict the frequencies to some algebraic variety. This happens, for example, when looking to the very classical results on analytic continuation, such as the Fabry gap theorem [14], when the functions to be studied are analytic in some half-plane, and have a representation of the form

$$
f(z)=\sum_{j=1}^{+\infty} c_{\jmath} \exp \left(i a_{\jmath} z\right),
$$

for $\left\{a_{j}\right\}$ a sequence of complex numbers. A similars ituation occurs in the study of Dirichlet series [6], which are again holomorphic in some half-plane, satisfy suitable growth conditions, and have a representation such as $(0.1)$; it is then clear that (in these cases) the sufficient set is a sequence $\left\{a_{j}\right\}$ of complex numbers and therefore far from being an algebraic variety in $C$.

The way Ehrenpreis dealt with these problems (in Part $C$ of [7]) was to consider $\left\{a_{j}\right\}$ as a sequence of algebraic varieties, and to try then to apply his results on such varieties.

Our approach in this paper (which we partially anticipated in a paper [2] where the simpler case of the Fabry gap theorem was dealt with), consists in considering $\left\{a_{j}\right\}$ as an analytic variety which arises as the zero set of an entire function satisfying suitable growth conditions (which are dictated by the growth of $\left\{a_{j}\right\}$ itself). In this framework, representations such as (0.1) are just a particular case of more general representations for solutions of convolution equations in spaces of holomorphic functions with growth conditions.

Let us now describe more precisely the contents of our paper. In Section 1 , we introduce the weight $p(z)=|z|^{\circ}, \rho>1$ and the space $A_{p .0}(\Gamma)$ which will be our main interest; for $\Gamma$ a cone in $C, A_{p, 0}(\Gamma)$ is the space of functions which are holomorphic in $\Gamma$, of order $\leqq \rho$ and minimal type (see (1.2) for the precise definition). Theorem 1.1 is a Paley-Wiener type theorem which characterizes the dual space $A_{p, 0}(\Gamma)^{\prime}$, identifying it with a space of entire functions whose growth is bounded by a weight which reflects the non-symmetry of $A_{p, 0}(\Gamma)$. By taking $\mu \in A_{p, 0}(\Gamma)^{\prime}, f \in A_{p, 0}(\Gamma)$ one can define $\mu * f$ which turns out to belong to a space $A_{p, 0}(\tilde{\Gamma})$, with $\tilde{\Gamma}$ a cone depending on $\Gamma$ and $\mu$; the chapter ends with Theorem 1.3, where it is shown that, under natural conditions, $\mu^{*}$ is a surjective operator. All of this is, so to speak, preliminary to section 2, where interpolation in $\left(A_{p, 0}(\Gamma)^{\prime}\right)^{\wedge}$ is studied. The main results of this section are Theorems 2.4 and 2.5 which completely describe the space of functions $f \in A_{p, 0}(\Gamma)$ such that $\mu * f=0$; in particular, Theorem 2.5 shows that such functions have the series representation (2.8) which naturally generalizes (0.1). Section 3 finally applies this whole theory to the study of Dirichlet series of the form 


$$
\sum_{n=1}^{+\infty} c_{n} \exp \left(-\lambda_{n} z\right), \quad\left\{\lambda_{n}\right\} \subseteq C
$$

In particular, Corollary 3.1 shows that (under natural hypotheses on $\left\{\lambda_{n}\right\}$ ) if a series such as (0.2) converges in some cone $\Gamma$ to a function in $A_{p, 0}(\Gamma)$, then it actually converges in $\boldsymbol{C}$ to an entire function in $A_{p, 0}(\boldsymbol{C})$. Deeper results (Theorem 3.3 and Corollary 3.2) give growth conditions on the coefficients $c_{n}$ which appear in (0.2). Our results, as it is clear, only hold for Dirichlet series whose frequencies $\left\{\lambda_{n}\right\}$ are zeros of functions in $\left(A_{p, 0}(\Gamma)^{\prime}\right)^{\wedge}$ and therefore we have a precise control on their density, in particular, it has not escaped our attention that even more interesting results could be obtained by replacing the weight $p(z)=|z|^{\rho}$ with the weight $p(z)=e^{|z|}$, as in this case the $\left\{\lambda_{n}\right\}$ might become more dense; for the time being however, we have not yet been able to deal with this case, as the interpolation theory would become, here, more complicated: we plan to return to this question in the near future. The last section of the paper deals with the problem of generalizing our method to the case of several variables. As we pointed out in [2], this question (in principle at least) does not give rise to new difficulties, but the details are not always easy to work out. In order to clarify the situation, we examine, in Section 4, the case of convolution equations in $H(\Omega)$ for $\Omega \leqq C^{n}$ an open convex set (i. e., we look at a particularly simple situation, from the point of view of the weights), and we prove a several variables version of the Fabry gap theorem and other related results.

Acknowledgement. The second author expresses his gratitude to the Department of Mathematics of the University of Maryland, where part of this work has been carried out.

\section{$\S 1$. Convolution in $A_{p, 0}(\Gamma)$ : Preliminaries}

In this section we develop some preparatory material on convolution equations in spaces of holomorphic functions with suitable growth conditions.

Generally speaking, these are problems which have already been studied in many different situations $[1],[3],[4],[5],[16],[17]$, but the specific spaces we have to deal with (and which originate while studying Dirichlet series) present some new difficulties which make it impossible for us to refer to any previous work.

Throughout the remainder of the paper, $\rho>1$ will be a real number, and $\sigma>1$ will be its conjugate, i.e., $\rho^{-1}+\sigma^{-1}=1 ; p=p(z)$ and $q=q(z)$ will, respectively, denote the subharmonic functions on $C, p(z)=|z|^{\rho}$ and $q(z)=|z|^{\sigma}$. To begin with some familiar spaces [4], [5], we will denote by $A_{p}(\boldsymbol{C})$ the space of all entire functions $f \in H(\boldsymbol{C})$ such that, for some $A, B>0$, 


$$
|f(z)| \leqq A \exp (B p(z)), \quad z \in C .
$$

This space (with its natural topology) is usually known under the name of the space of entire functions of order $\leqq \rho$ and finite type. On the other hand, one can also define the topological vector space $A_{p, 0}(\boldsymbol{C})$ of entire functions of order $\leqq \rho$ and minimal type, i. e., those functions $f \in H(C)$ such that $\forall \varepsilon>0, \exists A_{\varepsilon}>0$ such that

$$
|f(z)| \leqq A_{\varepsilon} \exp (\varepsilon p(z)), \quad z \in \boldsymbol{C} .
$$

An important relation can be seen to link all these definitions [18]; let $A_{p}(\boldsymbol{C})^{\prime}$ denote the strong dual space of $A_{p}(\boldsymbol{C})$, i.e., the space of continuous linear functionals on $A_{p}(\boldsymbol{C})$ endowed with the strong topology: one can then define the Laplace transform of a functional $\mu \in A_{p}(\boldsymbol{C})^{\prime}$ as the entire function

$$
\hat{\mu}(w)=\mu(z \longmapsto \exp (-z w)) \quad \text { for } z, w \in \boldsymbol{C},
$$

and it can be shown that (with the obvious meaning of the notations) [18]

and conversely

$$
\left(A_{p}(\boldsymbol{C})^{\prime}\right)^{\wedge}=A_{q, 0}(\boldsymbol{C})
$$

$$
\left(A_{p, 0}(\boldsymbol{C})^{\prime}\right)^{\wedge}=A_{q}(\boldsymbol{C})
$$

We now describe a considerably more complicated situation, which is the one we need to develop in order to study general Dirichlet series. Consider an open convex cone $\Gamma$, contained in $\Pi_{+}=\{z \in C: \operatorname{Re} z>0\}$ with vertex at the origin (this restriction, of course, is not necessary); for the sake of simplicity, we will suppose that $\Gamma=\{z \in C:-\psi<\arg z<\phi\}$, for some $\phi \in(0, \pi / 2]$ (so that $\Gamma=\Pi_{+}$for $\left.\phi=\pi / 2\right)$; we now denote by $A_{p, 0}(\Gamma)$ the space of all functions $f \in H(\Gamma)$ such that, for all $\varepsilon>0$ and for all cones $\Gamma^{\prime} \Subset \Gamma$, (by this we mean both that the vertex of $\Gamma^{\prime}$ is in $\Gamma$ as well as the opening of $\Gamma^{\prime}$ is strictly smaller than that of $\Gamma$ )

$$
|f(z)| \leqq C \exp (\varepsilon p(z)), \quad \text { for } z \in \Gamma^{\prime},
$$

for some constant $C=C\left(\varepsilon, \Gamma^{\prime}, f\right)>0$. We will endow $A_{p, 0}(\Gamma)$ with the projective limit topology which (1.2) naturally induces.

Our first objective is to find and prove the analogous to (1.1) for $A_{p, 0}(\Gamma)$. The first step, of course, consists in identifying the space $A_{p, 0}(\Gamma)^{\prime}$ of linear continuous functionals on $A_{p, 0}(\Gamma)$; we observe, first of all, that a linear continuous functional on $A_{p, 0}(\Gamma)$ can always be described by integration against a measurable function $u$, supported in some cone

$$
K=\left\{z \in \Pi_{+}:-\alpha \leqq \arg z \leqq \alpha\right\}+c,
$$

for some $c \in \mathbb{R}^{+}$and $\alpha \in(0, \phi)$, and such that, for some $A>0$,

$$
|u(z)| \leqq \text { const. } \exp (-A p(z)), \quad z \in K,
$$


$K$ is shown in the following picture:

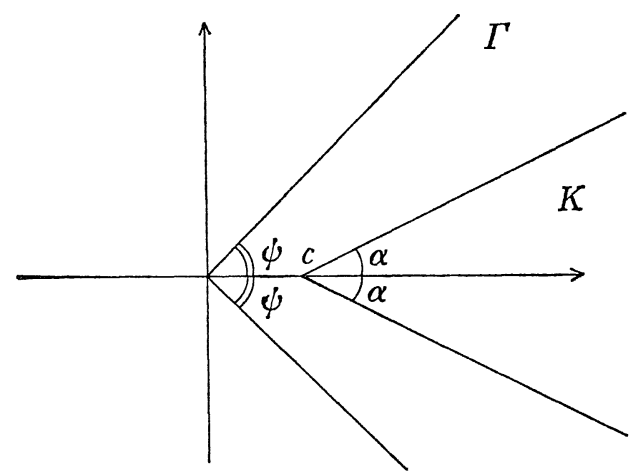

Therefore, to any $u \in A_{p, 0}(\Gamma)^{\prime}$ we can associate (in a non-unique way!) a value $c \in \boldsymbol{R}^{+}$and an angle $\alpha \in(0, \phi)$; if $u$ is supported by $K=K(\alpha, c)$, then $u$ is supported by any $K^{\prime}=K^{\prime}\left(\alpha^{\prime}, c^{\prime}\right)$ with $c^{\prime} \leqq c, \alpha \leqq \alpha^{\prime}$.

We now wish to define the Laplace transform of a functional $u \in A_{p, 0}(\Gamma)^{\prime}$; to do this, we first notice that the functions

$$
e_{w}(z)=\exp (-z \cdot w)
$$

belong (as functions of $z$ ) to $A_{p, 0}(\Gamma)$, for every choice of $w \in \boldsymbol{C}$; we therefore define the Laplace transform on $A_{p, 0}(\Gamma)^{\prime}$ by the following formula:

$$
\hat{u}(w)=\langle u, \exp (-z \cdot w)\rangle=\int_{C} u(z) \exp (-z \cdot w) d \lambda(z),
$$

where $d \lambda$ is the Lebesgue measure on $C$, i.e.,

$$
\hat{u}(w)=\int_{K} u(z) \exp (-z \cdot w) d \lambda(z) .
$$

Let us now write $w$, the dual variable of $z$, as $w=|w| \exp (i \varphi), \varphi \in[0,2 \pi)$, and define the following auxiliary function

$$
\beta(w)=\beta(\varphi ; \alpha)
$$

(which is a function of the argument of $w$ and also depends on the angle $\alpha$ ) as follows:

$$
\beta(\varphi ; \alpha)= \begin{cases}0 & 0 \leqq \varphi \leqq(\pi / 2)-\alpha \\ \cos (\pi-\alpha-\varphi) & (\pi / 2)-\alpha<\varphi \leqq \pi-\alpha \\ 1 & \pi-\alpha<\varphi \leqq \pi+\alpha \\ \cos (\pi+\alpha-\varphi) & \pi+\alpha<\varphi \leqq(3 \pi / 2)+\alpha \\ 0 & (3 \pi / 2)+\alpha \leqq \varphi \leqq 2 \pi\end{cases}
$$




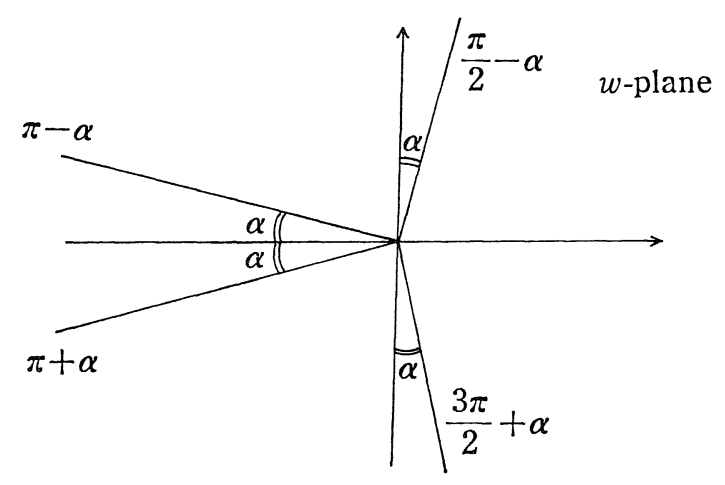

Theorem 1.1. $A_{p, 0}(\Gamma)^{\prime}$ is isomorphic, via the Laplace transform, to the space of entire functions $f \in H(C)$ such that, for some $B>0$ and some $\alpha \in(0, \phi)$, satisfy the inequality

$$
|f(w)| \leqq \text { const. } \exp \left(B|w|^{\sigma} \beta(\varphi ; \alpha)^{\sigma}-\frac{1}{B} \operatorname{Re}(w)\right) .
$$

We will denote by $\left(A_{p, 0}(\Gamma)^{\prime}\right)^{\wedge}$ this space.

Proof. Let $u \in A_{p, 0}(\Gamma)^{\prime}$, with $\operatorname{supp}(u) \subset K$, and consider its Laplace transform (1.4). By (1.3) we get (with a constant $A$ which depends on $u$ )

$$
|\hat{\imath}(w)| \leqq \text { const. } \exp \left(\max _{z \in K}\left(\operatorname{Re}(-z \cdot w)-A|z|^{\circ}\right)\right) .
$$

By the definition of $K$ one has $z=c+r e^{i \theta}$, with $-\alpha \leqq \theta \leqq \alpha$, hence

$$
\operatorname{Re}(-z \cdot w)=-[c|w| \cos \varphi+r|w| \cos (\theta+\varphi)] .
$$

where, of course, $\alpha$ depends on our choice of $K$, which in turn depends on $u$ (but always $\alpha \in(0, \psi)$ ). To compute the maximum in (1.6) we first estimate $\cos (\theta+\varphi)$. A simple computation shows that $-\cos (\theta+\varphi)<0$ for $0 \leqq \varphi<(\pi / 2)-\alpha$ or $(3 \pi / 2)+\alpha<\varphi \leqq 2 \pi$ while $\max _{\theta}(-\cos (\theta+\varphi))=\beta(\varphi ; \alpha)=\beta$ for all other values of $\varphi$. Therefore, the maximum to compute is given by

$$
\max _{r>0}\left\{r|w| \beta-A r^{\rho}-c|w| \cos \varphi\right\}
$$

Taking the derivative with respect to $r$ we get that the maximum takes place for $r$ such that

i.e.,

$$
|w| \beta-\rho A r^{\rho-1}=0
$$

$$
r=\left(\frac{|\omega| \beta}{\rho A}\right)^{1 /(\rho-1)}
$$


which gives as maximum value:

$$
\frac{|w|^{\sigma} \beta^{\sigma}}{A^{1 /(\rho-1)}}\left\{\left(\frac{1}{\rho}\right)^{1 /(\rho-1)}-\left(\frac{1}{\rho}\right)^{\sigma}\right\}-c \operatorname{Re}(w)
$$

with $\sigma=\frac{\rho}{\rho-1}>1$. That is,

$$
|\hat{\imath}(w)| \leqq \text { const. } \exp \left[c(\rho) \frac{|w|^{\sigma} \beta^{\sigma}}{A^{1 /(\rho-1)}}-c \operatorname{Re}(w)\right] .
$$

On the other hand, if $\beta=0$, the maximum is still given by the previous expression. Notice also that $c \operatorname{Re}(w)=c|w| \cos \varphi$ is positive when $\beta=0$, and so the estimate (1.5) follows by taking

$$
B=\max \left(\frac{c(\rho)}{A^{1 /(\rho-1)}}, \frac{1}{c}\right) .
$$

Of course, the bound in (1.7) is more precise, at it shows the relations between the bound of $\hat{u}$, those of $u$ and its support. We have, therefore, proved that $\left(A_{p, 0}(\Gamma)^{\prime}\right)^{\wedge}$ is contained in the space of entire functions with bounds given by (1.5).

To prove the converse, let $u \in A_{p, 0}(\Gamma)^{\prime}$ be represented by a measurable function on $\boldsymbol{R}^{2}$, which we still denote by $u$, supported by a suitable cone $K \subset \boldsymbol{R}^{2}$, and satisfying (1.3) in $K$. We can now define the Fourier transform of $u$ (as a function on $R^{2}$ ) by

$$
\mathscr{F} u(\eta, \zeta)=\int_{R^{2}} u(x, y) \exp (-x \eta-y \zeta) d \lambda(x, y) .
$$

In (1.8), the variables $x$ and $y$ are real variables, while $\eta$, $\zeta$ are complex variables, as $\mathcal{I} u$ is now defined on $C^{2}$ (while $\hat{u}$, the Laplace transform defined by (1.4), is defined on $C$ ); we remark that the integral in (1.8) is finite, as it only uses values $(x, y)$ in $K$, where $u$ decreases fast enough. An immediate direct computation shows that the Fourier transform of $u$ satisfies the following bound:

$$
|\mathscr{F} u(\eta, \zeta)| \leqq \text { const. } \exp \left(\max _{(x, y) \in K}\left\{\operatorname{Re}[-(x, y) \cdot(\eta, \zeta)]-A\left(x^{2}+y^{2}\right)^{\rho / 2}\right\}\right) .
$$

The usual Paley-Wiener type of argument shows that, indeed, (1.9) characterizes the space $\mathscr{F}\left(A_{p, 0}(\Gamma)^{\prime}\right)$ of those entire functions which are Fourier transforms (defined as in (1.8)) of elements of $A_{p, 0}(\Gamma)^{\prime}$. One can now see that the growth provided by (1.9) coincides, for $\zeta=i \eta$, with the growth given in (1.6), identifying $z$ with $(x, y)$ and $\left(w_{1}, w_{2}\right)$ with $\left(\eta_{1}, \eta_{2}\right)$. To conclude the proof of the theorem, it is now sufficient to consider an entire function $f \in H(\boldsymbol{C})$ satisfying (1.5) (or, equivalently, (1.6)); by using the standard Hörmander's extension result from algebraic varieties, (see e.g. [8]), we can extend $f$ from $C$ to an entire function $F \in H\left(C^{2}\right)$, by identifying $\boldsymbol{C} \subseteq C^{2}$ with the subvariety $\left\{(\eta, \zeta) \in C^{2}: \zeta=i n\right\}$, and $F$ 
satisfying the bounds (1.9). By applying the inversion theorem we just mentioned, $F=\mathscr{I} u$ for some $u \in A_{p, 0}(\Gamma)^{\prime}$, and therefore $f=\hat{u}$ for the same $u$.

Remark 1.1. i) Because of the construction we have followed, one can easily show that $|w|^{\sigma} \beta^{\sigma}$ is a subharmonic function.

ii) $\left(A_{p, 0}(\Gamma)^{\prime}\right)^{\wedge}$ is an algebra under pointwise multiplication.

We now proceed to the study of convolution equations in the spaces $A_{p, 0}(\Gamma)^{\prime}$ defined before; let $\mu \in A_{p, 0}(\Gamma)^{\prime}$ be represented by a measurable function $\varphi$ supported in some convex cone $K$, with vertex at $c$ and satisfying there the correct growth conditions : for $f \in A_{p, 0}(\Gamma)$ define

$$
\mu * f(x)=\int_{\Gamma} \varphi(x+t) f(t) d t
$$

In order for $x+t$ to remain in $K$ one needs, as $t \in \Gamma$, that $x$ be such that

$$
K-\{x\} \subseteq \Gamma,
$$

in other words,

$$
x \in c-\Gamma
$$

so that $\mu * f(x)$ is defined in $c-\Gamma$.

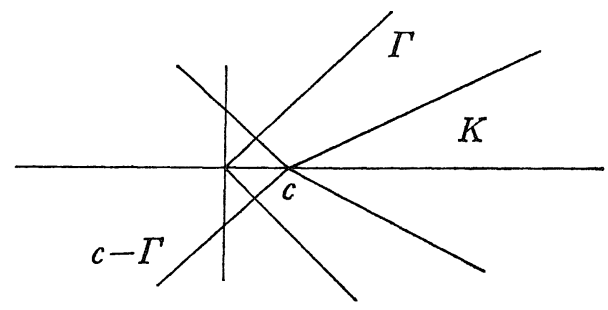

Note that, since

$$
\mu * f(x)=\int_{\Gamma} \varphi(x+t) f(t) d t=\int_{K} \varphi(y) f(y-x) d y,
$$

and since $\frac{\partial f}{\partial z}$ also belongs to $A_{p, 0}(\Gamma)$, we can take derivatives under the integral sign to deduce that $\mu * f \in H(c-\Gamma)$. An observation which will be needed in Section 3 (where we shall deal with Hamburger type theorems) is the following: if $f \in A_{p, 0}(\Gamma) \cap H(\boldsymbol{C})$, then $\mu * f$ can be defined as an entire function; indeed, for a fixed $x$ (or $x$ in some compact set) we can write $K=K_{0} \cup\left(K \backslash K_{0}\right)$, with $K_{0}$ bounded and such that the set $\left(K \backslash K_{0}\right)-x$ is still contained in $\Gamma$, so that

$$
\mu * f(x)=\int_{K_{0}} \varphi(y) f(y-x) d y+\int_{K \backslash K_{0}} \varphi(y) f(y-x) d y ;
$$

this shows that $\mu * f$ is well defined for 


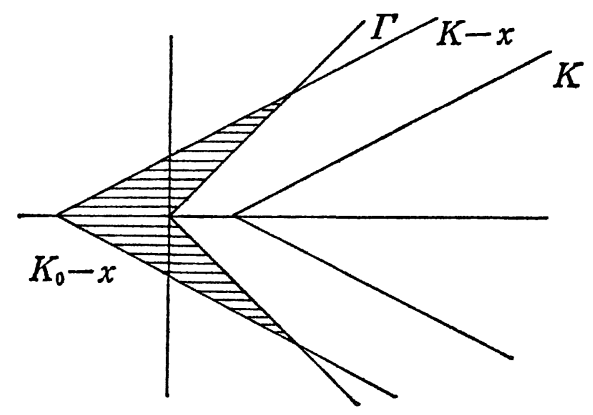

all $x \in \boldsymbol{C}$, since $f(y-x)$ is bounded on $K_{0}$ because $K_{0}$ is a bounded set, while no problems arise on $K \backslash K_{0}$ where $f$ satisfies the necessary growth conditions. Finally, it is easy to show that $\mu * f$ is an entire function as well.

We now investigate the growth of $\mu * f$, for $f \in A_{p, 0}(\Gamma)$ and $\mu \in A_{p, 0}(\Gamma)^{\prime}$. Let $\mu$ be represented by a measurable function $\varphi$, supported by some $K$ as before, and such that, on $K$,

$$
|\varphi(z)| \leqq A \exp \left(-B|z|^{\rho}\right)
$$

for some $B>0$ (notice that if (1.10) holds for some positive $B_{0}$, it then holds for all positive $B \leqq B_{0}$ ). Take now a cone $\Gamma^{\prime} \Subset \Gamma$, where, by (1.2), we have

$$
|f(z)| \leqq C \exp \left(\varepsilon|z|^{\rho}\right) ;
$$

we then obtain, for $x$ such that $K-\{x\} \cong \Gamma^{\prime}$,

$$
|\mu * f(x)| \leqq D \int \exp \left(-B|x+t|^{\rho}+\varepsilon|t|^{\rho}\right), \quad D=D\left(\varepsilon, \Gamma^{\prime}, f\right) .
$$

Since

one deduces that

$$
|t|^{\circ} \leqq 2^{\rho-1}\left(|x|^{\rho}+|t+x|^{\rho}\right)
$$

$$
-B|x+t|^{\rho}+\varepsilon|t|^{\rho} \leqq\left(\varepsilon-\frac{B}{2^{\rho-1}}\right)|t|^{\rho}+B|x|^{\rho} ;
$$

hence, by taking $\varepsilon<\frac{B}{2^{\rho-1}}$ (which is allowed in view of the projective limit structure of $\left.A_{p, 0}(\Gamma)\right)$, we get, for some $D^{\prime}=D^{\prime}\left(\varepsilon, \Gamma^{\prime}, f\right)>0$

$$
|\mu * f(x)| \leqq D^{\prime} \exp \left(B|x|^{\circ}\right),
$$

for all $x$ in $C$ such that $K-\{x\} \subset \Gamma^{\prime}$. Note that, in (1.11), the constant $B$ can be made as small as we wish and that, as $\Gamma^{\prime}$ exhausts $\Gamma$, the inequality (1.11) holds in a corresponding sequence of cones exhausting $c-\Gamma$; we have therefore shown that, for $\mu \in A_{p, 0}(\Gamma)^{\prime}$ :

$$
\mu *\left(A_{p, 0}(\Gamma)\right) \subseteq A_{p, 0}(c-\Gamma) .
$$


Remark 1.2. If we let $\mu^{*}$ act on $A_{p, 0}(\Gamma) \cap H(\boldsymbol{C})$, we will get, as a result, an entire function whose growth can be controlled (by the weight $p(z)$, but with different constants) on each translation (along the real axis) of the cone $c-\Gamma$.

To complete our preliminary study of convolution equations in $A_{p, 0}(\Gamma)$, we need to characterize the dual space of $A_{p, 0}(c-\Gamma)$; its elements are, of course, measurable functions $\varphi$, supported in some closed cone $K$ contained in the interior of $c-\Gamma$, with vertex on the real axis, and whose growth is bounded, on $K$ by $\exp (-B p(z))$, for some positive constant $B$. The space of their Laplace transforms is characterized by

Theorem 1.2. $A_{p, 0}(\Gamma)^{\prime}$ is isomorphic, via the Laplace transform, to the space of entire functions $f \in H(C)$ such that, for some $d, B>0$ and some $\alpha \in(0, \psi)$, satisfy an inequality of the form

$$
|f(w)| \leqq \text { const. } \exp \left[c(\rho) \frac{|w|^{\sigma} \beta^{\sigma}(\varphi+\pi ; \alpha)}{B^{1 /(\rho-1)}}+(c-d) \operatorname{Re}(w)\right] .
$$

This space of entire functions will be denoted by $\left(A_{p, 0}(c-\Gamma)^{\prime}\right)^{\wedge}$.

Proof. This result can be obtained as an immediate corollary of Theorem 1.1. Indeed, if $u \in A_{p, 0}(c-\Gamma)$, (we identify the functional and the measurable function which represents it) then it is immediate to verify that the function $f(z)$ defined by

$$
v(z)=u(-z-c)
$$

gives an element in $A_{p, 0}(\Gamma)^{\prime}$, and therefore (by (1.7), and for some $d>0$ depending on $K$ ),

$$
|\hat{v}(w)| \leqq \text { const. } \exp \left[c(\rho) \frac{|w|^{\sigma} \beta^{\sigma}}{B^{1 /(\rho-1)}}-d \operatorname{Re}(w)\right]
$$

But, by definition, and with $t=-z-c$

$$
\hat{v}(w)=\int u(-z-c) e^{-z w} d z=\int u(t) e^{c w} e^{t w} d t=e^{c w} \cdot \hat{u}(-w),
$$

from this we conclude that

$$
|\hat{u}(w)|=\left|\hat{v}(-w) e^{c w}\right|=|\hat{v}(-w)| e^{c \operatorname{Re}(w)} .
$$

Finally, since $\arg (-w)=\arg (w)+\pi$, we apply (1.7) to obtain the desired inequality.

According to the usual approach in the theory of convolution equations (see e.g., [4], [16]), we consider the transpose of the map 


$$
\mu^{*}: A_{p, 0}(\Gamma) \rightarrow A_{p, 0}(c-\Gamma)
$$

which is, again, a convolution

$$
\mu^{*^{\prime}}: A_{p, 0}(c-\Gamma)^{\prime} \rightarrow A_{p, 0}(\Gamma)^{\prime},
$$

defined, for $v \in A_{p, 0}(\Gamma)$ and $u \in A_{p, 0}(c-\Gamma)^{\prime}$, by

$$
\left\langle\mu^{*^{\prime}} u, v\right\rangle=\langle u, \mu * v\rangle=\int u(x)\left(\int \mu(x+t) v(t) d t\right) d x .
$$

Let us now take the Laplace transform of this map:

$$
\begin{aligned}
\left(\left(\mu^{*^{\prime}} u\right)^{\wedge}\right)(w) & =\int u(x)\left(\int \mu(x+t) e^{-t w} d t\right) d x \\
& =\int e^{-w(-x)} u(x)\left(\int \mu(x+t) e^{-(t+x)} w d t\right) d x \\
& =\hat{\mu}(w) \hat{u}(-w) .
\end{aligned}
$$

Now notice that, since $\hat{u}$ belongs to $A_{p, 0}(c-\Gamma)^{\prime}, \hat{u}(-w)$ has the growth which one would expect; to be more precise, from Theorems 1.1 and 1.2 one gets

$$
\begin{aligned}
& |\hat{\imath}(-w)| \leqq \text { const. } \exp \left[c(\rho) \frac{|w|^{\sigma} \beta^{\sigma}}{\beta^{1 /(\rho-1)}}+(d-c) \operatorname{Re}(w)\right], \\
& |\hat{\mu}(w)| \leqq \text { const. } \exp \left[c(\rho) \frac{|w|^{\sigma} \beta^{\sigma}}{A^{1 /(\rho-1)}}+c \operatorname{Re}(w)\right] .
\end{aligned}
$$

Hence, $\hat{u}(-w) \cdot \hat{\mu}(w)=\left(\left(\mu *^{\prime} u\right)^{\wedge}\right)(w)$ belongs to $\left(A_{p, 0}(\Gamma)^{\prime}\right)^{\wedge}$, and multiplication of $\hat{\mu}(w)$ induces a continuous map between

$$
\left(\left(A_{p, 0}(c-\Gamma)^{\prime}\right)^{\wedge}\right)^{\vee}=\left\{f(-w): f \in\left(A_{p, 0}(c-\Gamma)^{\prime}\right)^{\wedge}\right\}
$$

and $\left(A_{p, 0}(\Gamma)^{\prime}\right)^{\wedge}$.

We can now deal with the problem of surjectivity of such convolution operators :

Definition 1.1. Let $\mu \in A_{p, 0}(\Gamma)^{\prime}$ have a support in $K=\left\{z \in C: z=c+r e^{i \theta}\right.$ : $-\alpha \leqq \theta \leqq \alpha\}$ for some $c \in \boldsymbol{R}^{+}$and $\alpha \in(0, \psi)$. Then $\mu$ is said to be $(p, \Gamma)$-slowly decreasing if, for any $\varepsilon>0$, there exists a sequence of $\left\{r_{n}\right\}_{n=1}^{+\infty}, r_{n} \nearrow+\infty$, with $r_{n+1} \leqq A \cdot r_{n}$ for some $A>1$, and a constant $D>0$ such that

$$
|\hat{\mu}(w)| \geqq D \exp (-\varepsilon|w|),
$$

on the boundary of each of the following regions:

$$
H_{n}^{(\alpha)}=\left\{w \in C: r_{n} \leqq|w| \leqq r_{n+1}\right\} \cap H^{(\alpha)},
$$

$H^{(a)}=\{w \in C: 0 \leqq \arg w \leqq(\pi / 2)-\alpha\} \cup\{w \in C:(3 \pi / 2)+\alpha \leqq \arg w \leqq 2 \pi\}$. More simply, one may ask that $\forall \varepsilon>0, \exists\left\{r_{n}\right\}$ as above, such that the components of 
$\{w:|\hat{\mu}(w)| \leqq D \exp (-\varepsilon|w|)\}$ do not cross the boundaries of $H_{n}^{(\alpha)}$.

Remark 1.3. A simple class of examples for Definition 1.1 can be built with the use of infinite products. Let $z_{n}$ be a sequence of complex numbers such that $z_{n} \neq 0, \lim _{n \rightarrow+\infty} n / z_{n}=0, z_{-n}=-z_{n}$, and such that there exists a strictly positive constant $M>0$ such that $\left|z_{n}-z_{m}\right| \geqq M|n-m|$ (for any two integers $n, m)$; it is well known (see e.g., [14] thm. XXXI) that, under these conditions $f(\zeta)=\prod_{n=1}^{+\infty}\left(1+\frac{\zeta^{2}}{z_{n}^{2}}\right)$ is a well defined entire function of exponential type zero, i. e., for any $\varepsilon>0$ there exists $C_{\varepsilon}>0$ such that

$$
|f(\zeta)| \leqq C_{\varepsilon} \exp (\varepsilon|\zeta|) \text {. }
$$

Observe that $f$ is the Laplace transform of an analytic functional $\mu$ carried by the origin, so that $\mu^{*}$ acts on $A_{p, 0}(\Gamma)$ as an infinite order linear differential operator with constant coefficients (in this case $K=\{0\}$, so that both $c$ and $\alpha$ vanish). Now, the theory of infinite products (see e.g., [13]) shows that $f(\zeta)$ satisfies (1.12) everywhere, except on small neighborhoods (of diameters decreasing to zero) of the points $z_{n}$; this shows that $\mu$ is slowly decreasing according to our Definition 1.1.

The interest of Definition 1.1 lies in the following:

Theorem 1.3. If $\mu \in A_{p, 0}(\Gamma)^{\prime}$ is $(p, \Gamma)$-slowly decreasing, then

$$
\mu *\left(A_{p, 0}(\Gamma)\right)=A_{p, 0}(c-\Gamma),
$$

i.e., $\mu^{*}$ is surjective.

Proof. Standard functional analysis shows that we must prove that $\hat{\mu} \cdot\left(A_{p, 0}(c-\Gamma)^{\prime}\right)^{\wedge}$ is closed in $\left(A_{p, 0}(\Gamma)^{\prime}\right)^{\wedge}$; therefore it suffices to show that whenever $f \in\left(A_{p, 0}(\Gamma)^{\prime}\right)^{\wedge}$ and $f / \hat{\mu}$ is entire, then $f / \hat{\mu} \in\left(A_{p, 0}(c-\Gamma)^{\prime}\right)^{\wedge}$. To do this we first remark that both $f$ and $\hat{\mu}$ have exponential growth $\sigma$ and finite type, and so (by a variation of the Lindelöf theorem) $f / \hat{\mu}$ is, as well, of exponential growth $\sigma$ and finite type. We now use the fact that, on $H^{(\alpha)}$,

$$
|f(w)| \leqq \text { const. } \exp (-c \operatorname{Re}(w)) \sim \text { const. } \exp (-c|w|)
$$

while (for $d>0$ depending on the support of $\mu$ )

$$
|\hat{\mu}(w)| \leqq \text { const. } \exp [(d-c) \operatorname{Re}(w)] \sim \text { const. } \exp ((d-c)|w|),
$$

so that, choosing $\varepsilon<c$, we get that, outside each $H_{n}^{(\alpha)}$,

$$
|f(w) / \hat{\mu}(w)| \leqq \text { const. } \exp \left(-c^{\prime}|w|\right),
$$

for some $c^{\prime}>0$. Applying the maximum modulus theorem, the same estimate can be obtained inside $H_{n}^{(\alpha)}$, so that (1.13) actually holds on $H^{(\alpha)}$, with $f / \hat{\mu}$ 
which is (globally) of exponential growth $\sigma$ and finite type. To prove that $f / \hat{\mu}$ has the growth prescribed for the elements in $\left(A_{p, 0}(c-\Gamma)^{\prime}\right)^{\wedge}$, we now take $\alpha^{\prime}>\alpha$, so that $\mu$ is certainly supported in a cone $K^{\prime}, K^{\prime} \Subset \Gamma$ of opening $\alpha^{\prime}$ and of vertex $c-\varepsilon$ (for $\varepsilon$ sufficiently small). We now see that, on $H^{\left(\alpha^{\prime}\right)}, f / \hat{\mu}$ is bounded by const. $\exp \left(-c^{\prime}|w|\right)$, as we wanted to show. The same estimate holds in $H^{(\alpha)} \backslash H^{\left(\alpha^{\prime}\right)}$, and it is certainly smaller than const. $\exp \left(c(\rho)|w|^{\sigma} \beta^{\sigma}\right)$. Finally no problem arises outside $H^{(\alpha)}$; this proves the theorem.

\section{$\S 2$. Convolution in $A_{p, 0}(\Gamma)$ : Interpolation}

In this second section we deal with the more sophisticated aspects of the theory of convolution equations in $A_{p, 0}(\Gamma)$, namely those which are related to interpolation problems.

In what will follow, we shall assume $\mu \in A_{p, 0}(\Gamma)^{\prime}$, and, for $D>0$ and $\varepsilon>0$, we will denote

$$
\begin{array}{r}
S(\hat{\mu} ; \varepsilon, D)=\left\{w \in C:|\hat{\mu}(w)| \leqq D \exp (-\varepsilon|w|) \text { if } w \in H^{(\alpha)}\right. \\
\left.|\hat{\mu}(w)| \leqq D \exp \left(-\varepsilon|w|^{\sigma}\right) \quad \text { otherwise }\right\} .
\end{array}
$$

All other notations remain unchanged. Our first basic tool is the following semi-local to global interpolation result:

Theorem 2.1. Let $\varepsilon>0$ be sufficiently small, $D>0$, and let $\tilde{\lambda}$ be a function analytic on $S(\hat{\mu} ; \varepsilon, D)$ such that, for some constants $C, B>0$,

$$
|\tilde{\lambda}(w)| \leqq C \exp \left(B|w|^{\sigma} \beta(w)^{\sigma}-\frac{1}{B} \operatorname{Re}(w)\right) \quad \text { on } S(\hat{\mu} ; \varepsilon, D) \text {. }
$$

Then there exists $\lambda \in\left(A_{p, 0}(\Gamma)^{\prime}\right)^{\wedge}$ and $\gamma$ holomorphic on $S\left(\hat{\mu} ; \varepsilon^{\prime}, D^{\prime}\right)$ such that

$$
\lambda(w)=\tilde{\lambda}(w)+\gamma(w) \hat{\mu}(w) \text { on } S\left(\hat{\mu} ; \varepsilon^{\prime}, D^{\prime}\right)
$$

and

$$
|\gamma(w)| \leqq C^{\prime} \exp \left(B^{\prime}|w|^{\sigma} \beta(w)^{\sigma}-\left(1 / B^{\prime}\right) \operatorname{Re}(w)\right) \text { on } S\left(\hat{\mu} ; \boldsymbol{\varepsilon}^{\prime}, D^{\prime}\right) .
$$

Proof. Even though the proof follows well known lines, it is worthwhile repeating it, in view of some differences with other related results. First, one can choose $\varepsilon^{\prime}<\varepsilon, D^{\prime}>D$ and $\chi \in C^{\infty}(C)$ so that $0 \leqq \chi \leqq 1, \quad \chi=1$ on $S\left(\hat{\mu} ; \varepsilon^{\prime}, D^{\prime}\right) \subset S(\hat{\mu}, \varepsilon, D), \chi$ has support in $S(\hat{\mu} ; \varepsilon, D)$ and

$$
|(\partial \chi / \partial \bar{z})(w)| \leqq A \exp \left(\Theta_{B}(w)\right)
$$

where we denoted

$$
\Theta_{B}(w)=B|w|^{\sigma} \beta(w)^{\sigma}-\frac{1}{B} \operatorname{Re}(w) .
$$

Notice that the possibility of obtaining (2.2) depends on the fact that, separately, $|w|^{\sigma} \beta(w)^{\sigma}$ and $|w|$ are subharmonic functions, and that $\boldsymbol{C}$ can be divided ints 
sectors in which either one dominates. Then, both $\chi \tilde{\lambda}$ and $[\partial(\chi \tilde{\lambda}) / \partial \bar{z}] / \hat{\mu}$ are both $C^{\infty}$ functions (since $\bar{\partial}(\chi \tilde{\lambda})$ vanishes on $S\left(\hat{\mu} ; \varepsilon^{\prime}, D^{\prime}\right)$ ) and (for $\varepsilon^{\prime}$ small enough with respect to $1 / B$ )

$$
|[\partial(\chi \tilde{\lambda}) / \partial \bar{z}] / \hat{\mu}| \leqq A^{\prime} \exp \left(\Theta_{B^{\prime}}(w)\right) .
$$

By applying the $L^{2}$-methods of Hörmander [8], one finds $\gamma \in C^{\infty},|\gamma(w)| \leqq$ $A^{\prime \prime} \exp \left(\Theta_{B^{\prime \prime}}(w)\right)$ such that $\partial \gamma / \partial \bar{z}=\partial(\chi \tilde{\lambda}) / \partial \bar{z}$, and the theorem now follows by taking

since

$$
\lambda=\chi \tilde{\lambda}-\gamma \hat{\mu}
$$

$$
\frac{\partial \lambda}{\partial \bar{z}}=\frac{\partial}{\partial \bar{z}}(\chi \tilde{\lambda})-\frac{\partial \gamma}{\partial \bar{z}} \hat{\mu}=0,
$$

while (2.1) implies both that $\lambda \in\left(A_{p, 0}(\Gamma)^{\prime}\right)^{\wedge}$ and that the estimates on $\gamma$ hold. (Note that $\gamma$ is, in fact, analytic in $S\left(\hat{\mu}, \varepsilon^{\prime}, D^{\prime}\right)$ ).

Remark 2.1. Theorem 2.1 is weaker than the usual semilocal to global interpolation theorem. This is due to the fact that, in $S(\hat{\mu} ; \varepsilon, D)$, we do not use $\Theta_{B}(w)$, but only a part of it; this, however, is of no concern, as for slowly decreasing convolutors small values only have to controlled on $H^{(\alpha)}$, where $-|w|$ and $\Theta_{B}(w)$ essentially agree. The importance of this fact will be clear in the sequel.

We now recall some basic definitions, which we adapt to our situation: in the sequel $V=\left\{\left(z_{k}, m_{k}\right)\right\}$ will be a multiplicity variety in $C$, i. e., $\left\{z_{k}\right\}$ is a discrete set of points which do not accumulate anywhere, and $m_{k}$ is an integer bigger than or equal to one. It is clear that, for any entire function $f \in H(\boldsymbol{C})$, $f$ defines a multiplicity variety $V(f)$, where the $z_{k}$ are the zeros of $f$ and $m_{k}$ represents the multiplicity of $z_{k}$ as a zero of $f$ : conversely, the most elementary interpolation theorem (Weierstrass Theorem) shows that for every multiplicity variety $V$, there always exists $f \in H(\boldsymbol{C})$ such that $V=V(f)$. If $V=\left\{\left(z_{k}, m_{k}\right)\right\}$ is a multiplicity variety, we can consider the ideal

$$
\begin{aligned}
I=I(V)= & \left\{F \in H(\boldsymbol{C}): F \text { vanishes at } z_{k}\right. \text { with multiplicity } \\
& \text { bigger than or equal to } \left.m_{k}\right\},
\end{aligned}
$$

and clearly two functions $h, g \in H(\boldsymbol{C})$ can be identified modulo $I$ if and only if

$$
\frac{1}{l !} \frac{d^{l} h}{d z^{l}}\left(z_{k}\right)=\frac{1}{l !} \frac{d^{l} g}{d z^{l}}\left(z_{k}\right)=a_{k, l}, \quad 0 \leqq l<m_{k}, \quad k=1,2, \cdots .
$$

It can be shown, moreover, that given any sequence $\left\{a_{k, l}\right\}$ with $k=1,2, \cdots$, $0 \leqq l<m_{k}$, there exists $F \in H(\boldsymbol{C})$ such that its $l$-th Taylor coefficient at $z_{k}$ is $a_{k, l}$, so that $H(C) / I$ can be identified to the space of all sequences $\left\{a_{k, l}\right\}$; we will denote (as customary) $H(\boldsymbol{C}) / I$ by $H(V)$, and the natural map

$$
\rho_{V}=\rho: H(\boldsymbol{C}) \rightarrow H(V)
$$


which associates to an entire function $F$, its equivalence class modulo $I$ or, equivalently, the sequence $\left\{\frac{1}{l !} \frac{d^{l} F}{d z^{l}}\left(z_{k}\right)\right\}$ of its Taylor coefficients, will be called restriction map.

It is our purpose to study the image, under the map $\rho$, of the space $\left(A_{p, 0}(\Gamma)^{\prime}\right)^{\wedge} \subset H(\boldsymbol{C})$, when $V$ is the multiplicity variety of $\hat{\mu}$, for some $\mu \in A_{p, 0}(\Gamma)^{\prime}$, and to discuss some related interpolation problems.

Definition 2.1. Let $V=\left\{\left(z_{k}, m_{k}\right)\right\}$ be a multiplicity variety. The space

$$
\left(A_{p, 0}(\Gamma)^{\prime}\right)^{\wedge}(V)
$$

is defined to be the space of all functions $\left\{a_{k, l}\right\} \in H(V)$ such that, for some $C, B>0$,

$$
\sum_{l=0}^{m_{k}-1}\left|a_{k, l}\right| \leqq C \exp \left(\Theta_{B}\left(z_{k}\right)\right), \quad k=1,2, \cdots
$$

Remark 2.2. When the multiplicities satisfy good bounds, i.e., when

$$
m_{k}=\mathcal{O}\left(\exp \left(\Theta_{B}\left(z_{k}\right)\right)\right), \quad k=1,2, \cdots
$$

for some $B>0$, then (2.3) is equivalent to the simpler

$$
\left|a_{k, l}\right| \leqq C_{1} \exp \left(\Theta_{B_{1}}\left(z_{k}\right)\right), \quad k=1,2, \cdots
$$

for some $C_{1}, B_{1}>0$.

Remark 2.3. It is clear, from the definition of $\Theta_{B}(w)$, that $\rho\left(\left(A_{p, 0}(\Gamma)^{\prime}\right)^{\wedge}\right) \subseteq$ $\left(A_{p, 0}(\Gamma)^{\prime}\right)^{\wedge}(V)$.

Definition 2.2. $\quad V$ will be called an interpolating variety (with respect to $\left.\left(A_{p, 0}(\Gamma)^{\prime}\right)^{\wedge}\right)$ if

$$
\rho\left(\left(A_{p, 0}(\Gamma)^{\prime}\right)^{\wedge}\right)=\left(A_{p, 0}(\Gamma)^{\prime}\right)^{\wedge}(V) .
$$

Remark 2.4. Characterizations of interpolating varieties for a wide class of weights are well known whenever $\Gamma=\boldsymbol{C},[4],[12]$, but this seems to be the first paper (together with [2]) in which the case of a cone $\Gamma \varsubsetneqq \boldsymbol{C}$ is considered.

Theorem 2.2. Let $f \in\left(A_{p, 0}(\Gamma)^{\prime}\right)^{\wedge}$ be $(p, \Gamma)$-slowly decreasing with multiplicity variety $V=\left\{\left(z_{k}, m_{k}\right)\right\}$ and suppose that, for $\varepsilon>0$ sufficiently small, and some $D>0$, the components of the set $S(f ; \varepsilon, D)$ contain at most one point of $V$. Then $V$ is interpolating.

Proof. The first step is to recall that, with the notations of Section 1, $f \in A_{q}(\boldsymbol{C})$ and that (see [4]) every non-zero function is slowly decreasing in $A_{q}(\boldsymbol{C})$. Therefore, in view of our hypotheses, and of Corollary 2 in $[4], V(f)$ is interpolating in the sense of $A_{q}(\boldsymbol{C})$. Therefore, if $\left\{a_{k, l}\right\} \in\left(A_{p, 0}(\Gamma)^{\prime}\right)^{\wedge}(V) \subseteq A_{q}(V)$, 
there exists $F \in A_{q}(\boldsymbol{C})$ such that $\rho_{V}(F)=\left\{a_{k, l}\right\}$. Clearly $F$ does not necessarily belong to $\left(A_{p, 0}(\Gamma)^{\prime}\right)^{\wedge}$, but we can use it to produce the function we need. For this purpose, consider the components $U_{j}$ of $S(f ; \varepsilon, D)$; notice that since $f$ is ( $p, \Gamma$ )-slowly decreasing, the components $U_{j}$ do not intersect the boundaries of the regions $H^{(\alpha)}$ associated to $\varepsilon, D$. We now define $\tilde{\lambda}$ on $S(f ; \varepsilon, D)$ as follows: if $U_{j} \cap H^{(\alpha)}=\varnothing, \tilde{\lambda}=\left.F\right|_{U_{j}}$, while if $U_{j} \subset H^{(\alpha)}$, we have two possibilities: if there exists $z_{k} \in U_{j}$, we set

$$
\lambda(z)=\sum_{i=0}^{m_{k}-1} a_{k, i}\left(z-z_{k}\right)^{i}
$$

(by hypothesis, no other points of $V$ are in $U_{j}$ ), while we set $\tilde{\lambda} \equiv 0$ if $U_{j} \cap V=\varnothing$. The function $\tilde{\lambda}$ which we have constructed in this way clearly satisfies the right bounds, and therefore the theorem follows immediately from Theorem 2.1.

Remark 2.5. As in Remark 1.3, infinite products can be used to provide concrete examples of interpolating varieties.

The same argument employed in the proof of Theorem 2.2, together with some geometric results of W. A. Squires ([15]), shows that we can rephrase differently our result, to provide a more geometric sufficient condition for interpolation:

Theorem 2.3. Let $f \in\left(A_{p, 0}(\Gamma)^{\prime}\right)^{\wedge}$ be $(p, \Gamma)$-slowly decreasing with multiplicity variety $V=\left\{\left(z_{k}, m_{k}\right)\right\}$, and let $n\left(z_{k}, t\right)$ be the number of points of $V \backslash\left\{z_{k}\right\}$ whose distance from $z_{k}$ is less than or equal to $t$. Suppose that, for some $\varepsilon>0$ sufficiently small and some $D>0$, the components of the sets $H_{n}^{(\alpha)}$ contain at most one point of $V$, and that there are constants $A, B>0$ such that

$$
\begin{aligned}
& \int_{0}^{\left|z_{k}\right|} n\left(z_{k}, t\right) \frac{d t}{t} \leqq A\left|z_{k}\right|^{\sigma}+B, \\
& m_{k} \leqq \frac{A\left|z_{k}\right|^{\sigma}+B}{\log \left|z_{k}\right|},
\end{aligned}
$$

then $V$ is interpolating.

Proof. As in Theorem 2.2, this follows from Theorem 2.1, after applying Theorem 3 of [15], instead of Corollary 2 of [4].

We can now proceed to the main results of this section which concern mean-periodic functions in $A_{p, 0}(\Gamma)$. Given $\mu \in A_{p, 0}(\Gamma)^{\prime}$ and $f \in A_{p, 0}(\Gamma)$, we shall say that $f$ is $\mu$-mean periodic (or, simply, mean-periodic if no confusion can arise) if $\mu * f$ vanishes identically on $c-\Gamma$; we will now try to follow the lines of [4], [5] to describe the space of mean-periodic functions. In order to do this, it is necessary to group the points in $V=\{z \in C: \hat{\mu}(z)=0\}$ which fall in 
the same connected component of $S(\hat{\mu} ; \varepsilon, D)$. Let then $\left\{a_{k, l}\right\} \in\left(A_{p, 0}(\Gamma)^{\prime}\right)^{\wedge}(V)$, and consider a representative $\varphi \in H(C)$ of it. If we denote by $\left\{U_{\alpha}\right\}_{\alpha}$ the connected components of $S(\hat{\mu} ; \varepsilon, D)$, we know by Lindelöf's Theorem and Definition 1.1 they are bounded and with diameters satisfying good bounds. One can construct analytic functions $\varphi_{\alpha} \in H\left(U_{\alpha}\right)$ by setting (for $z \in U_{\alpha}$ )

$$
\varphi_{\alpha}(z)=\frac{1}{2 \pi i} \int_{\partial U_{\alpha}} \frac{\varphi(\zeta)}{\hat{\mu}(\zeta)} \cdot \frac{\hat{\mu}(\zeta)-\hat{\mu}(z)}{\zeta-z} d \zeta
$$

It is immediate to verify that, for $V_{\alpha}=V \cap U_{\alpha}$,

$$
\rho_{V_{\alpha}}\left(\varphi_{\alpha}\right)=\rho_{V_{\alpha}}(\varphi)=\left\{a_{k, l}^{(\alpha)}\right\}=\left\{a_{k, l}: z_{k} \in V_{\alpha}\right\},
$$

and that $\varphi_{\alpha}$ does not depend on the choice of the representative $\varphi$ of $\left\{a_{k, l}\right\}$ (all of this follows from the Cauchy formula).

Definition 2.3. $\hat{A}_{p, 0, g, \Gamma}(V)$ is the space of those functions

$$
\left\{a_{k, l}\right\} \in\left(A_{p, 0}(\Gamma)^{\prime}\right)^{\wedge}(V)
$$

such that, for every $\alpha$

$$
\left\|a_{k, l}^{(\alpha)}\right\|_{\alpha}:=\inf \left\{\|\varphi\|_{\infty}: \varphi \in H\left(U_{\alpha}\right), \rho_{V_{\alpha}}(\varphi)=\left\{a_{k, l}^{(\alpha)}\right\}\right\} \leqq A \exp \left(\Theta_{B}(z)\right)
$$

for some $A, B>0$ and all $z \in U_{\alpha}$ (observe that the constants $A, B$ do not depend on $\alpha$ ). The norms defined by (2.5) on $H\left(U_{\alpha}\right)$ clearly determine a topological structure on $\hat{A}_{p, 0, g, \Gamma}(V)$.

Theorem 2.4. (Fundamental Principle). If $\mu$ is ( $p, \Gamma$ )-slowly decreasing, the map $\rho_{V}$ induces a linear topological isomorphism between

$$
\left(A_{p, 0}(\Gamma)^{\prime}\right)^{\wedge} /\left(\hat{\mu} \cdot\left(A_{p, 0}(c-\Gamma)^{\prime}\right)^{\wedge}\right)
$$

and $\hat{A}_{p, 0, g, \Gamma}(V)$.

Proof. First, it is trivial to show that if $\left\{a_{k, l}\right\}=\rho_{V}(\varphi)$ for some $\varphi \in\left(A_{p, 0}(\Gamma)^{\prime}\right)^{\wedge}$, then, there exist $A, B>0$, such that for $\varepsilon>0$ small enough

$$
\left|\varphi_{\alpha}(z)\right| \leqq A \exp \left(\Theta_{B}(z)\right), \quad z \in U_{\alpha} .
$$

On the other hand, using the hypothesis that $\mu$ is $(p, \Gamma)$-slowly decreasing, one can show that, given $A_{1}, B_{1}>0$, there are $A, B$ such that

$$
\left\|a_{k, l}^{(\alpha)}\right\|_{\alpha} \leqq A_{1} \exp \left(\Theta_{B_{1}}(z)\right) \text { on } U_{\alpha}
$$

implies (2.6). Conversely, if (2.6) holds, then (2.7) also holds, for suitable values of $A_{1}, B_{1}>0$. A standard application of Theorem 2.1 concludes the proof.

Remark 2.6. As the reader can see, this theorem is the analogue, in our slightly more complicated situation, of the well known Fundamental Principle 
of Ehrenpreis [7] and of its many generalizations to convolution equations [1], $[3],[5],[16]$.

The reason for the interest in Theorem 2.4 is due to the following crucial representation theorem for mean periodic functions in $A_{p, 0}(\Gamma)$ :

Theorem 2.5. (Representation theorem). If $f \in A_{p, 0}(\Gamma)$ is $\mu$-mean-periodic for some $\mu \in A_{p, 0}(\Gamma)^{\prime}$, and $\mu$ is $(p, \Gamma)$-slowly decreasing, then $f$ has a Fourier representation, convergent in the topology of $A_{p, 0}(\Gamma)$, as follows:

$$
f(w)=\sum_{\alpha}\left(\sum_{\left(z_{k}, l\right) \in V_{\alpha}} c_{k, l} w^{l} \exp \left(-z_{k} \cdot w\right)\right), \quad w \in \Gamma .
$$

Proof. Since $\mu$ is $(p, \Gamma)$-slowly decreasing, $\hat{\mu} \cdot\left(A_{p, 0}(c-\Gamma)^{\prime}\right)^{\wedge}$ is closed in $\left(A_{p, 0}(\Gamma)^{\prime}\right)^{\wedge}$ (Theorem 1.3) and therefore the space

$$
\left\{f \in A_{p, 0}(\Gamma): \mu * f=0\right\}
$$

is the dual space to $\left(A_{p, 0}(\Gamma)^{\prime}\right)^{\wedge} / \hat{\mu} \cdot\left(A_{p, 0}(c-\Gamma)^{\prime}\right)^{\wedge}$ which, by Theorem 2.4, is isomorphic to $\hat{A}_{p, 0, g, \Gamma}(V)$. Now, an element $F \in\left[\hat{A}_{p, 0, g, \Gamma}(V)\right]^{\prime}$ is just a sequence of functionals $\left\{F_{\alpha}\right\}, F_{\alpha} \in\left[H\left(V_{\alpha}\right)\right]^{\prime}$ whose norms are such that, for any $\varphi \in \hat{A}_{p, 0, g, \Gamma}(V)$, the series

$$
F(\varphi)=\sum_{\alpha} F_{\alpha}\left(\varphi_{\alpha}\right)
$$

would converge. Therefore, given a mean periodic function $f$, we associate to it a functional $F \in\left[\hat{A}_{p, 0, g, \Gamma}(V)\right]^{\prime}$ which is related to $f$ by (see Theorem 2.4)

$$
\nu(f)=f\left(\rho_{V}(\hat{\nu})\right),
$$

for any $\nu \in A_{p, 0}(\Gamma)^{\prime}$. We can now take for any $w \in \Gamma, \nu=\delta_{w}, \hat{\nu}(z)=\exp (-z w)$, and so, the representation (2.8) follows immediately from (2.9).

Remark 2.7. The groupings in (2.8) are a consequence of the fact that each open set $U_{\alpha}$ might contain more than one zero of $\hat{\mu}$; in many interesting cases, however, this does not happen (see e.g., Theorem 2.2), and whenever each open set $U_{\alpha}$ contains at most a single zero of $\hat{\mu}$, the representation (2.8) can be simplified, to

$$
f(w)=\sum_{k} c_{k}(w) \exp \left(-z_{k} w\right),
$$

where $c_{k}$ is a polynomial of degree less than $m_{k}$. Whenever a representation such as (2.10) is possible for the $\mu$-mean-periodic functions of $A_{p, 0}(\Gamma)$ (even if $U_{\alpha}$ contains more than one zero of $\hat{\mu}$ ), we shall say (in analogy with what happens in simpler cases [4]) that $V$ is weak interpolating.

We conclude this section with two corollaries of (the proof of) Theorem 2.5: 
Corollary 2.1. Let $f \in A_{p, 0}(\Gamma)$ be $\mu$-mean periodic for some ( $p, \Gamma$ )-slowly decreasing $\mu \in A_{p, 0}(\Gamma)^{\prime}$. Then the constants $c_{k, l} \in C$ which appear in the Fourier representation (2.8) of $f$ satisfy the following estimate:

$$
\left|c_{k, l}\right|=\mathcal{O}\left(\exp \left(-\Theta_{B}\left(z_{k}\right)\right)\right)
$$

for any $B>0$, as $k \rightarrow \infty$.

Corollary 2.2. For $f$ as in Corollary 2.1, the coefficients $c_{k, l}$ are uniquely determined by $f$ itself.

Remark 2.8. Both Corollaries 2.1 and 2.2 are well known if $\Gamma=C$, see e.g., [4]. On the other hand, Corollary 2.2 is not generally true when in $\boldsymbol{C}^{n}, n>1$ (even in very simple cases) due to the more difficult (and not unique) definition of multiplicity variety of a system of homogeneous convolution equations.

\section{§3. Dirichlet Series}

The motivations for the results contained in this chapter come from the classical book [6] by V. Bernstein on Dirichlet series, as well as from Chapter XII of L. Ehrenpreis' treatise [7].

Consider, indeed, a Dirichlet series

$$
\sum_{n=1}^{+\infty} c_{n} e^{-\lambda_{n}^{n}}, \quad \lambda_{n} \in C
$$

as it is well known, [6], such a series admits, as a convergence domain, a half plane of the form $\{z \in C: \operatorname{Re} z>a\}$, with $a$ any real number (or possibly, $a= \pm \infty$, in which case the series is, respectively, nowhere or everywhere convergent). In its convergence domain, the sum of the series is a holomorphic function $f$ which may, however, be analytically continued outside this convergence domain. In this case, a basic problem in the theory of Dirichlet series consists in determining the existence (and possibly the distribution) of the singular points of $f$. It turns out immediately, however, that the study of the existence of singular points for $f$ is strongly dependent on the "density" of the exponents $\left\{\lambda_{n}\right\}$; let us recall at this moment that a sequence $\left\{\lambda_{n}\right\}$ is said to be measurable of density $D$ if

$$
\lim _{n \rightarrow+\infty} \frac{n}{\lambda_{n}}=D
$$

and is said to be of maximum density $D$ if it is a subsequence of a measurable, density $D$ sequence (without being, of course, a subsequence of a smaller density sequence); in all other cases, one says that $\left\{\lambda_{n}\right\}$ has infinite density. In particular, whenever the sequence $\left\{\lambda_{n}\right\}$ has finite density, V. Bernstein obtained quite general results [6], which did not need any a priori knowledge on the coefficients 
$c_{n}$; it is not without interest to remark that the methods employed by Bernstein strongly rely on the possibility of interpolating the coefficients of the series by analytic functions (this is somehow reminiscent of our own approach). On the other hand, no general results could be obtained by V. Bernstein for the case of infinite density, and very restrictive conditions had to be introduced. This problem was one of the motivations for our work, as it is clear if one recalls the relations which link the growth of our entire functions and the density of the sequence of its zeros. These relations are carefully described in [6] and [13], but let us just recall that if $\left\{\lambda_{n}\right\}$ is a measurable sequence with density $D$, then there exists an entire function $f$, which vanishes exactly at the points $z= \pm \lambda_{n}$, and such that

$$
|f(z)| \leqq A_{\varepsilon} \exp [(D+\varepsilon)|z|], \quad z \in \boldsymbol{C} .
$$

If, on the other hand, we look for functions in spaces such as $A_{p, 0}(\boldsymbol{C})$, then we see that their zeros form sequences of infinite density. It is therefore clear that our results establish a first systematic attempt to deal with the problem of singularities of analytic functions associated with infinite density sequences. Let us conclude this introductory comment by pointing out that we do not deal here with all the interesting sequences of infinite density. The main example of our shortcomings arises if one considers the sequence

$$
\lambda_{n}=\log n \text {. }
$$

Such a sequence has infinite density, and it is of great interest as it gives rise to the classical Dirichlet series of the kind

$$
\sum_{n=1}^{+\infty} c_{n} n^{-s}
$$

i. e., ultimately to the Riemann $\zeta$-function. The reason is that a sequence such as $\{-\log n\}$ arises from the zeros of faster growing functions than those considered in this paper. Namely functions whose growth is bounded by $e^{e^{|z|}}$ and the interpolation theory with respect to this growth has not yet been completely worked out.

We remind the reader that besides the classical Dirichlet series we are simultaneously considering the case of "generalized" Dirichlet series where the coefficients can be polynomials.

Definition 3.1. A sequence $\left\{\lambda_{n}\right\}$ of complex numbers is said to be strictly $(p, \Gamma)$-slowly decreasing if there exists $\mu \in A_{p, 0}(\Gamma)^{\prime}, \mu(p, \Gamma)$-slowly decreasing, such that $Z(\hat{\mu})=\left\{\lambda_{n}\right\}$.

Definition 3.2. A sequence $\left\{\lambda_{n}\right\}$ as above is said to be weakly $(p, \Gamma)$-slowly decreasing if there exists $\mu \in A_{p, 0}(\Gamma)^{\prime}, \mu(p, \Gamma)$-slowly decreasing, such that $Z(\hat{\mu}) \supseteqq\left\{\lambda_{n}\right\}$. 
Definition 3.3. Let $\left\{\left(\lambda_{n}, m_{n}\right)\right\}$ be a multiplicity variety; we say that $\left\{\left(\lambda_{n}, m_{n}\right)\right\}=V$ is strictly $(p, \Gamma)$-slowly decreasing (resp. weakly $(p, \Gamma)$-slowly decreasing) if there exists $\mu \in A_{p, 0}(\Gamma)^{\prime}, \mu(p, \Gamma)$-slowly decreasing, such that $V=Z(\hat{\mu})$ (resp. $V \subseteq Z(\hat{\mu}))$.

Remark 3.1. Definition 3.3 contains Definitions 3.1 and 3.2 as special cases, when $m_{n}=1$ for all $n$.

Remark 3.2. Examples of sequences (and multiplicity varieties) which are strictly and weakly $(p, \Gamma)$-slowly decreasing can be immediately obtained from Remarks 1.3 and 2.5 .

Theorem 3.1. Let $\mu \in A_{p, 0}\left(\Pi_{+}\right)^{\prime}$ be $\left(p, \Pi_{+}\right)$-slowly decreasing. Suppose that, for some $\theta \in(0, \pi / 2)$, the intersection

$$
Z_{\theta}(\hat{\mu})=Z(\hat{\mu}) \cap\{w \in C:-\theta<\arg w<\theta\}
$$

contains finitely many points. Then every $f \in A_{p, 0}\left(\Pi_{+}\right)$solution of $\mu * f=0$ extends to some $\tilde{f} \in A_{p, 0}(\boldsymbol{C})$ which satisfies the same equation.

Proof. We apply a well known argument concerning comparison theorems for convolution equations as in [2]. Since $\mu$ is slowly decreasing and $\mu * f=0$, $f$ can be represented as an element of the dual space

$$
\left(\frac{\left(A_{p, 0}\left(\Pi_{+}\right)^{\prime}\right)^{\wedge}}{\hat{\mu} \cdot\left(A_{p, 0}^{\prime}\left(\Pi_{-}+c\right)^{\prime}\right)^{\wedge}}\right)^{\prime}
$$

$c>0$ depending on $\mu$, and $\Pi_{-}=\{z \in C: \operatorname{Re} z<0\}$. In ${ }_{i=1}^{*}$ view of Theorem 2.4, this last space is isomorphic to the space:

$$
\hat{A}_{p, 0, g, \Pi_{+}}(V)
$$

of functions on the multiplicity variety $V, V=Z(\hat{\mu})$, whose growth is bounded by $\tilde{p}(w)=|w|^{\sigma} \beta(w)^{\sigma}-c \operatorname{Re}(w)$ where we can choose $\beta$ so that it vanishes in a cone around $\operatorname{Im}(w)=0$, completely contained in $\{-\theta<\arg w<\theta\}$, as we did in the proof of Theorem 1.3 ; but since $Z_{\theta}(\hat{\mu})$ only consists of finitely many points, and since on $Z(\hat{\mu}) \backslash Z_{\theta}(\hat{\mu})$ one has that $\tilde{p}$ and $q=|w|^{\sigma}$ are comparable (i. e., for some $A>0, \tilde{p} \leqq q \leqq A \tilde{p})$, one deduces that, actually $f$ represents an element in $A_{q}(V)$. Since every $\mu \in A_{p, 0}\left(\Pi_{+}\right)^{\prime}$ belongs to $A_{p, 0}(\boldsymbol{C})^{\prime}$ and it is slowly decreasing in this space, the space $A_{q}(V)$ is isomorphic to the space of $\mu$-mean-periodic functions in $A_{p, 0}$. Therefore $f$ admits an extension $\tilde{f}$, thereby concluding the proof of the theorem.

Remark 3.3. This result has an obvious translation in terms of generalized Dirichlet series with groupings. Indeed, by Theorem 2.5 , every $f \in A_{p, 0}\left(\Pi_{+}\right)$ which is a solution of $\mu * f=0$ can be represented by a series, converging in 
$A_{p, 0}\left(\Pi_{+}\right)$, of the type

$$
\sum_{n=1}^{+\infty} \sum_{j=1}^{N_{n}} c_{n, \jmath}(z) \exp \left(-\lambda_{n, j} z\right)
$$

where $Z(\hat{\mu})=\left\{\left(\lambda_{n}, m_{n}\right)\right\}$; hence Theorem 3.1 says that every generalized Dirichlet series as in (3.1), convergent in $A_{p, 0}\left(\Pi_{+}\right)$, does actually converge in $A_{p, 0}(\boldsymbol{C})$, provided that $\left\{\left(\lambda_{n}, m_{n}\right)\right\}$ is a strictly $\left(p, \Pi_{+}\right)$-slowly decreasing multiplicity variety which, for some $\theta \in(0, \pi / 2)$ intersects $\{w \in C:-\theta<\arg w<\theta\}$ in at most a finite number of points.

Theorem 3.1 can be immediately extended to the case of functions in $A_{p, 0}(\Gamma), \Gamma \subseteq \Pi_{+}$as follows:

Theorem 3.2. Let $\mu \in A_{p, 0}(\Gamma)$ be ( $\left.p, \Gamma\right)$-slowly decreasing and such that, for some $\theta \in\left(\frac{\pi}{2}-\psi, \pi / 2\right), Z_{\theta}(\hat{\mu})$ contains only finitely many points. Then every $\mu$ mean periodic function $f$ in $A_{p, 0}(\Gamma)$ extends to some $\tilde{f} \in A_{p, 0}(\boldsymbol{C})$, still $\mu$-mean periodic.

Corollary 3.1. Let $\left\{\left(\lambda_{n}, m_{n}\right)\right\}$ be a strictly $(p, \Gamma)$-slowly decreasing multiplicity variety, with (except for a finite number of indices $n$ )

$$
\arg \left(\lambda_{n}\right) \notin\left(-\theta+\phi-\frac{\pi}{2}, \theta-\phi+\frac{\pi}{2}\right),
$$

for some $\theta \in(0, \pi / 2)$; then any generalized Dirichlet series convergent in $\Gamma$,

$$
\sum_{n=1}^{+\infty} \sum_{j=1}^{N} c_{n, j}(z) \exp \left(-\lambda_{n, j} z\right)
$$

actually converges in $\boldsymbol{C}$, to an entire function in $A_{p, 0}(\boldsymbol{C})$.

We now proceed to prove a deeper form of Theorems 3.1 and 3.2, in which the $\lambda_{n}$ are allowed to be weakly slowly decreasing and, most important, can be chosen also in $R^{+}$.

Theorem 3.3. Let $\left\{\lambda_{n}\right\}$ be a weakly $\left(p, \Pi_{+}\right)$-slowly decreasing sequence of complex numbers, associated to some $\mu \in A_{p, 0}\left(\Pi_{+}\right)^{\prime}$ such that $Z(\hat{\mu})$ is a weak interpolating variety. Consider the series

$$
\sum_{n=1}^{+\infty} c_{n} e^{-\lambda_{n} z}
$$

which we assume to be convergent in $\Pi_{+}$, to a holomorphic function $f$. Suppose, moreover, that $f$ extends to an entire function $\tilde{f} \in A_{p, 0}(\boldsymbol{C})$. Then

$$
c_{n}=\mathcal{O}\left(\exp \left(-B\left|\lambda_{n}\right|^{\sigma}\right)\right), \quad \forall B>0 \text {. }
$$


Proof. Since $\left\{\lambda_{n}\right\}$ is weakly $\left(p, \Pi_{+}\right)$-slowly decreasing, we can find $\mu \in A_{p, 0}\left(\Pi_{+}\right)^{\prime}$ which is $\left(p, \Pi_{+}\right)$-slowly decreasing, and such that $Z(\hat{\mu}) \supseteqq\left\{\lambda_{n}\right\}$. Since $f \in A_{p, 0}\left(\Pi_{+}\right), \mu * f$ is a well defined holomorphic function in some half plane $\Pi_{-}+c(c>0$ depending on $\mu)$. From the representation (3.2) of $f$ in $\Pi_{+}$ we deduce that $\mu * f$ actually vanishes in $\Pi_{-}+c$. Now consider the extension $\tilde{f} \in A_{p, 0}(\boldsymbol{C})$ of $f$; since $\boldsymbol{\mu} \in A_{p, 0}\left(\Pi_{+}\right)^{\prime} \subset A_{p, 0}(\boldsymbol{C})^{\prime}, \mu * \tilde{f}$ is a well defined entire function which, by analytic continuation, is identically zero. We can therefore use Remark 2.6 to show that $\tilde{f}$ is represented in $\boldsymbol{C}$ by a series

$$
\tilde{f}(z)=\sum_{j=1}^{+\infty} d_{j} \exp \left(-w_{j} z\right)
$$

(no groupings are necessary, in view of the hypotheses on $\hat{\mu}$; besides, we assume here that all zeros $w_{j}$ of $\hat{\mu}$ are simple zeros). Now, Theorem 2.6 (actually Remark 2.8) immediately implies the bound

$$
d_{j}=\mathcal{O}\left(\exp \left(-B\left|w_{j}\right|^{\sigma}\right)\right), \quad \forall B>0 .
$$

We now see, however, that $f=\left.\tilde{f}\right|_{\Pi_{+}}$, and therefore $f$ has two series representations in $\Pi_{+}$, as a mean-periodic function in $A_{p, 0}\left(\Pi_{+}\right)$. From Theorem 2.7 we conclude that (3.3) holds.

Remark 3.4. Theorem 3.3 immediately extends to the case in which a cone $\Gamma \leqq \Pi_{+}$is used to replace $\Pi_{+}$.

Theorems 3.2 and 3.3 yield the following.

Corollary 3.2. Let $\mu \in A_{p, 0}(\Gamma)^{\prime}$ be $(p, \Gamma)$-slowly decreasing, and suppose that $\hat{\mu}$ has only simple zeros. If $Z(\hat{\mu})=\left\{\lambda_{n}\right\}$ is weakly $(p, \Gamma)$-interpolating and $Z_{\theta}(\hat{\mu})$ contains finitely many points for some $\theta \in\left(\frac{\pi}{2}-\psi, \pi / 2\right)$, then, every series

$$
\sum_{n=1}^{+\infty} c_{n} e^{-\lambda} n^{z}
$$

uniformly convergent on compact subsets of $\Gamma$ satisfies the bounds

$$
c_{n}=\mathcal{O}\left(\exp \left(-B\left|\lambda_{n}\right|^{\sigma}\right)\right), \quad \forall B>0 .
$$

\section{§4. Several Variables}

In Remark 11 of [2], we pointed out that our interpolation methods on analytic varieties in $\boldsymbol{C}$ can be (at least in principle) extended to discrete analytic varieties in $\boldsymbol{C}^{n}$; this remark also applies to our approach for Dirichlet series, but since no detail was given in [2], we have included in this section a more precise treatment. For the sake of simplicity, we will deal with holomorphic functions with no growth conditions, as this will save us the effort of develop- 
ing, from scratch, the several variables version of Section 1; as a corollary we will be able to prove a several variables version of the Fabry gap theorem (see also [2], [7], [10], [11]), which is of remarkable independent interest.

Let then $\Omega \subseteq C^{n}$ be a convex set; a rather complete theory of convolution equations in $H(\Omega)$ is developed in [17]; let therefore $\mu \in H^{\prime}\left(\boldsymbol{C}^{n}\right)$ be an analytic functional, carried by some compact convex set $K \subset C^{n}$, and let $\tilde{\Omega}$ be a convex subset of $\boldsymbol{C}^{n}$ such that $\tilde{\Omega}+K=\Omega$. Then $\mu$ acts as a convolutor

$$
\mu^{*}: H(\Omega) \rightarrow H(\tilde{\Omega})
$$

by associating to each $f \in H(\Omega)$ the analytic function

$$
\mu * f(z)=\langle\mu, \zeta \mapsto f(z+\zeta)\rangle .
$$

As in Section 1 we define the Laplace transform $\hat{\mu}$ of $\mu$ by

$$
\hat{\mu}(z)=\langle\mu, \zeta \mapsto \exp (-z \cdot \zeta)\rangle,
$$

which induces a continuous multiplicator

$$
\hat{\mu}:\left(H^{\prime}(\tilde{\Omega})\right)^{\wedge} \rightarrow\left(H^{\prime}(\Omega)\right)^{\wedge},
$$

between the spaces of entire functions $\left(H^{\prime}(\tilde{\Omega})\right)^{\wedge}$ and $\left(H^{\prime}(\Omega)\right)^{\wedge}$. The description of these spaces is well known (see Chapter $\mathrm{V}$ of [7]) and, if we denote by $H_{S}(z)=\sup _{\zeta \in S} \operatorname{Re}(z \cdot \zeta)$, the supporting function of a compact convex set $S$, we have:

$\left(H^{\prime}(\tilde{\Omega})\right)^{\wedge} \cong\left\{F \in H\left(C^{n}\right):\right.$ there exist $A>0$ and a convex compact $S \subset \tilde{\Omega}$, such that for all $\left.z \in \boldsymbol{C}^{n},|F(z)| \leqq A \exp \left(H_{S}(z)\right)\right\}$,

and

$$
\begin{gathered}
\left(H^{\prime}(\Omega)\right)^{\wedge} \cong\left\{F \in H\left(C^{n}\right): \text { there exist } A>0 \text { and a convex compact } S \subset \tilde{\Omega},\right. \\
\text { such that for all } \left.z \in C^{n},|F(z)| \leqq A \exp \left(H_{S+K}(z)\right)\right\} .
\end{gathered}
$$

In [17] it is shown that if $\mu_{1}, \cdots, \mu_{r} \in H^{\prime}\left(\boldsymbol{C}^{n}\right), 1 \leqq r \leqq n$, are carried by the same compact set $K$, and if their Fourier-Borel transforms satisfy suitable hypotheses, then one can prove a representation theorem for all functions $f \in H(\Omega)$ such that $\mu_{1} * f=\cdots=\mu_{r} * f=0$. Such a representation states that each such $f$ can be written as a convergent series (with suitable groupings, as in Section 3) of integrals of exponential solutions, and the integration is carried on the analytic variety $V=\left\{z \in \boldsymbol{C}^{n}: \hat{\mu}_{1}(z)=\cdots=\hat{\mu}_{r}(z)=0\right\}$. Our interest here is only the case when $V$ is a discrete variety, which occurs (in view of some natural regularity conditions on $\mu_{1}, \cdots, \mu_{r}$ ) when $r=n$. This case is simple enough to be completely described in here.

Definition 4.1. An $n$-tuple $\mu=\left(\mu_{1}, \cdots, \mu_{n}\right)$ of analytic functionals in $H^{\prime}\left(\boldsymbol{C}^{n}\right)$ carried by $K$ is said to be slowly decreasing with respect to $(\Omega, \tilde{\Omega})$ if there exists a constant $c>0$ and $m \in N$ such that, for all $z \in C^{n}$, 


$$
\left|\hat{\mu}_{i}(z)\right| \geqq c\left(\tilde{d}\left(z, V_{i}\right)\right)^{m} \exp \left(H_{K}(z)\right)(1+|z|)^{-m}, \text { for } i=1, \cdots, n,
$$

where $V_{\imath}=\left\{z \in \boldsymbol{C}^{n}: \hat{\mu}_{i}(z)=0\right\}$ and $\tilde{d}\left(z, V_{i}\right)=\min \left(1\right.$, distance from $z$ to $\left.V_{\imath}\right)$,

$$
\left(\sum_{i=1}^{n}\left|\hat{\mu}_{i}(z)\right|^{2}\right)^{1 / 2}=|\hat{\mu}(z)| \geqq c(\tilde{d}(z, V))^{m} \exp \left(H_{K}(z)\right)(1+|z|)^{-m},
$$

and for every $\varepsilon>0$ there are constants $A, B>0$ such that the set

$$
S(\hat{\mu} ; \varepsilon, A)=\left\{z \in C^{n}: \tilde{d}\left(z, V_{\imath}\right) \leqq A \exp (-\varepsilon|z|), 1 \leqq i \leqq n\right\}
$$

has relatively compact connected components, and for any $z_{1}$ and $z_{2}$ belonging to the same component of $S(\hat{\mu} ; \varepsilon, A)$, we have

$$
H_{S}\left(z_{1}\right) \leqq H_{S}\left(z_{2}\right)+B
$$

for all compact convex sets $S \subset \tilde{\Omega}$.

Remark 4.1. It can be shown, [1], [17], that most $n$-tuples of exponential sums satisfy the hypotheses of Definition 4.1 .

The interest of Definition 4.1 lies in the following results [17].

Theorem 4.1. (Fundamental Principle). If $\mu=\left(\mu_{1}, \cdots, \mu_{n}\right)$ is slowly decreasing, there is a linear topological isomorphism between

$$
\left(H^{\prime}(\Omega)\right)^{\wedge} /\left(\hat{\mu}_{1}\left(H^{\prime}(\tilde{\Omega})\right)^{\wedge}+\cdots+\hat{\mu}_{n}\left(H^{\prime}(\tilde{\Omega})\right)^{\wedge}\right)
$$

and a suitably defined subspace $\left(H^{\prime}(\Omega)\right)^{\wedge}(V)$ of the restriction $\rho_{V}\left(\left(H^{\prime}(\Omega)\right)^{\wedge}\right)$ to: $V$.

Theorem 4.2. (Representation Theorem). If $\mu=\left(\mu_{1}, \cdots, \mu_{n}\right)$ is slowly :decreasing, then every function $f \in H(\Omega)$ solution of the system $\mu_{1} * f=\cdots=\mu_{n} * f=0$ can be represented in the form

$$
f(z)=\sum_{k=1}^{+\infty}\left(\sum_{j=1}^{J_{k}} c_{k, j}(z) \exp \left(-\alpha_{k, j} \cdot z\right)\right), \quad z \in \Omega,
$$

where $V=\left\{z \in \boldsymbol{C}^{n}: \hat{\mu}_{1}(z)=\cdots=\hat{\mu}_{n}(z)=0\right\}=\bigcup_{k=1}^{+\infty}\left\{\alpha_{k, \jmath}: j=1, \cdots, J_{k}\right\}$, and where the polynomials $c_{k, j}$ are such that the functions $c_{k, j}(z) \exp \left(-\alpha_{k, j} \cdot z\right)$ are solutions of the given system.

We shall now apply the preceding theory to some particular choices-of $\Omega$. Indeed, we shall consider the convex subsets of $\boldsymbol{C}^{n}$ given by

and

$$
\Pi_{+}^{n}=\left\{z=\left(z_{1}, \cdots, z_{n}\right) \in \boldsymbol{C}^{n}: \operatorname{Re} z_{j}>0 \text { for any } j=1, \cdots, n\right\}
$$

$$
\Omega_{\theta}=\left(\Gamma_{\theta}\right)^{n},
$$

where $\Gamma_{\theta}$ is the subset of $C$ defined by 


$$
\Gamma_{\theta}=\{z \in C:-\theta<\arg z<\theta\}
$$

Remark 4.2. In the classical Fabry gap theorem (see also [2]) one considers, in $\boldsymbol{C}$, the upper half plane and not, as we are doing, the righth alf plane. We have, however, preferred this choice for the sake of uniformity with the previous sections of this paper.

We now give a simple preliminary lemma, in the spirit of Lemma 3 in [2]:

Lemma 4.1. Let $\mu=\left(\mu_{1}, \cdots, \mu_{n}\right)$ be a slowly decreasing $n$-tuple as above. Suppose that $\mu$ satisfies the following condition:

$\left(C_{\theta}\right)$ there exists $\varepsilon>0$ such that (with at most a finite number of exceptions) no $z \in V$ belongs to

$$
\begin{gathered}
\Omega_{\theta}^{*}=\left\{z \in C^{n}, z=\left(z_{1}, \cdots, z_{n}\right): \text { for some index } j\right. \\
\text { one has } \left.\frac{\pi}{2}+\theta-\varepsilon<\arg z_{3}<3 \frac{\pi}{2}-\theta+\varepsilon\right\} .
\end{gathered}
$$

Then, for any $\overrightarrow{\mathrm{a}}=(\mathrm{a}, \mathrm{a}, \cdots, \mathrm{a}) \in\left(\boldsymbol{R}^{+}\right)^{n}$, every solution $f \in H\left(\Omega_{\theta}-\overrightarrow{\mathrm{a}}\right)$ of $\mu_{1} * f=\cdots=$ $\mu_{n} * f=0$ uniquely extends to a solution $\tilde{f} \in H\left(\Pi_{+}^{n}-\vec{a}\right)$ of the same system.

Proof. Since $\mu$ is a slowly decreasing $n$-tuple and $\mu * f=0$, one deduces, with the help of Theorem 4.2, a series representation for $f$ itself, which converges on $\Omega_{\theta}-\overrightarrow{\mathrm{a}}$. Therefore (essentially, this is Theorem 4.1) if on $V$ the weights which describe the topologies of $\left(H^{\prime}\left(\Omega_{\theta}-\overrightarrow{\mathrm{a}}\right)\right)^{\wedge}$ and of $\left(H^{\prime}\left(\Pi_{+}^{n}-\overrightarrow{\mathrm{a}}\right)\right)^{\wedge}$ are comparable, one immediately concludes that the series representation of $f$ converges in $H\left(\Pi_{+}^{n}-\vec{a}\right)$ and the conclusion of the lemma follows. But, in view of the description of $\left(H^{\prime}(\Omega)\right)^{\wedge}$ which we have provided before, Condition $\left(C_{\theta}\right)$ actually implies such a result.

As an almost immediate corollary we get

Theorem 4.3. Let $\mu=\left(\mu_{1}, \cdots, \mu_{n}\right)$ be slowly decreasing, and suppose it satisfies $\left(C_{\theta}\right)$. Let $f \in H\left(\Omega_{\theta}\right)$ be a solution of $\mu_{1} * f=\cdots=\mu_{n} * f=0$. If there exists a neighborhood $U$ of the origin to which $f$ extends holomorphically, then we can find $\delta>0$ such that $f$ extends holomorphically to $\tilde{f} \in H\left(\Pi_{+}^{n}-\vec{\delta}\right)$, and such that there, $\mu_{1} * \tilde{f}=\cdots=\mu_{n} * \tilde{f}=0$.

Proof. The result follows exactly with the same argument we used to prove Theorem 1 in [2].

Remark 4.3. Theorem 4.3 immediately reduces, for $n=1$, to Theorem 1 in [2] and, therefore, provides the required extension of the Fabry gap theorem to several variables. 
Remark 4.4. In [10], a different extension of the Fabry gap theorem is discussed, and, in [2], we employ it to provide a strengthening of Theorem 4.3 , in which infinitely many points of $V$ are allowed in $\Omega_{\theta}^{*}$, provided they give rise to a suitable infinite order differential operator. Since, in [10], Kawai also gives a several variables version of his result, one might use Theorem 2 in [10], our Theorem 4.3 and the methods of Theorem 2 in [2], to provide a similar extension of Theorem 4.3. We leave the straightforward details to the reader.

Let us finally conclude with some considerations which turn out to be of some interest. The set $\Omega_{\theta}$ which we have employed provides, without any doubt, the correct generalization of the role played by $\Gamma_{\theta}$ in $C$ but, for many purposes, is not a very natural set in $\boldsymbol{C}^{n}$. A different approach consists in taking, e.g., an open convex cone $\Gamma \subset \boldsymbol{R}^{n}$, with vertex at the origin, and defining the tube over $\Gamma$ as $T_{\Gamma}=\boldsymbol{R}^{n}+i \Gamma$, then $T_{\Gamma}$ is an open convex subset of $\boldsymbol{C}^{n}$ to which, therefore, we can apply the theory described in Theorems 4.1 and 4.2 .

If now $\Gamma_{1} \subseteq \Gamma_{2}$ are two such cones, we can replace Condition $\left(C_{\theta}\right)$ with the (necessarily less specific) condition

$$
\begin{aligned}
& \left(C_{\Gamma_{1}, \Gamma_{2}}\right) \text { for any compact set } \tilde{K} \subset \Gamma_{2} \text {, there exists a compact set } K \subset \Gamma_{1}, \\
& \text { such that (with at most a finite number of exceptions), } \\
& \text { all } z \in V \text { satisfy } \sup _{\zeta \in K}[(\operatorname{Im} z) \cdot(\operatorname{Im} \zeta)] \leqq \sup _{\zeta \in \tilde{K}}[(\operatorname{Im} z) \cdot(\operatorname{Im} \zeta)] .
\end{aligned}
$$

The same arguments used in Lemma 4.1 and Theorem 4.3 are now sufficient to prove the following variation of Theorem 4.3.

Theorem 4.4. Let $\mu=\left(\mu_{1}, \cdots, \mu_{n}\right)$ be slowly decreasing (with respect to $\left.\left(T_{\Gamma_{1}}, T_{\Gamma_{2}}\right)\right)$, and suppose it satisfies $\left(C_{\Gamma_{1}, \Gamma_{2}}\right)$. Let $f \in H\left(T_{\Gamma_{1}}\right)$ be a solution of $\mu_{1} * f=\cdots=\mu_{n} * f=0$. If there is a neighborhood $\mathcal{U}$ of the origin to which $f$ extends holomorphically, then there exists a $>0$ such that $f$ extends holomorphically to $\tilde{f} \in H\left(T_{\Gamma_{2}-\overrightarrow{\mathrm{a}}}\right)$ in such a way that $\mu_{1} * \tilde{f}=\cdots=\mu_{n} * \tilde{f}=0$.

Actually, it is not difficult to give a hyperfunction version (see [9] for the standard notations) of this last result, which should justify our new approach:

Theorem 4.5. Let $\mu=\left(\mu_{1}, \cdots, \mu_{n}\right)$ be slowly decreasing and suppose it satisfies $\left(C_{\Gamma_{1}, \Gamma_{2}}\right)$. Let $f \in H\left(T_{\Gamma_{1}}\right)$ and denote by $\varphi(x)=f\left(x+i \Gamma_{1} 0\right)$ the hyperfunction defined on $\boldsymbol{R}^{n}$ by taking the boundary value of $f$ along $\Gamma_{1}$. If $f$ is a solution of $\mu_{1} * f=\cdots=\mu_{n} * f=0$ then the singularity spectrum of $\varphi$, s. s. $\varphi$, is contained in $\boldsymbol{R}^{n}+i \Gamma_{\frac{1}{2}}, \Gamma_{\frac{1}{2}}^{\frac{1}{2}}$ denoting the dual cone of $\Gamma_{2}$, namely,

$$
\Gamma_{\frac{1}{2}}=\left\{\eta \in \boldsymbol{R}^{n}: \xi \cdot \eta \geqq 0 \text { for all } \xi \in \Gamma_{2}\right\} .
$$


Proof. By the representation $\varphi(x)=f\left(x+i \Gamma_{1} 0\right)$, and Theorem 2.3.5 in [9], one has that s.s. $\varphi$, i. e., the support of the microfunction $\operatorname{sp}(\varphi)$, spectrum of $\varphi$, is contained in $\boldsymbol{R}^{n}+i \Gamma_{1}^{\frac{1}{1}}$. However, from the slow decrease of $\mu$ and the $\left(C_{\Gamma_{1}, \Gamma_{2}}\right)$ condition, one deduces that $f$ extends to $\tilde{f} \in H\left(T_{\Gamma_{2}}\right):$ just apply the arguments in Lemma 4.1. Since $\Gamma_{1} \subset \Gamma_{2}$, one has that $\varphi(x)=f\left(x+i \Gamma_{1} 0\right)=\tilde{f}\left(x+i \Gamma_{2} 0\right)$ and therefore, as before, s.s. $\varphi \subset \boldsymbol{R}^{n}+i \Gamma_{\frac{1}{2}}$ (notice that $\Gamma_{1} \subset \Gamma_{2}$ implies $\Gamma_{2}^{\perp} \subset \Gamma_{1}^{\perp}$, so that we have an improvement on the microanalyticity of $\varphi$ ).

\section{§5. Errata}

At this point, we wish to take the chance to correct a few misprints which can cause confusion in the reader of [2]:

a) page 571 , third line from the bottom should read:

"...by those $z$ which lie between the normals..."

b) page 573 , fourth line from the top should read:

"... is satisfied except for a countable..."

\section{References}

[1] Berenstein. C. A. and Struppa, D.C., Solutions of convolution equations in convex sets, Amer. J. Math., 109 (1987), 521-544.

[2] - On the Fabry-Ehrenpreis-Kawai gap theorem, Publ. RIMS, Kyoto Univ., 23 (1987), 565-574.

[3] - book in preparation.

[4] Berenstein, C.A. and Taylor, B. A., A new look at interpolation theory for entire functions of one variable, Advances in Math., 33 (1979), 109-143.

[5] - Interpolation problems in $C^{n}$ with applications to harmonic analysis, $J$. Anal. Math., 38 (1980), 188-254.

[6] Bernstein, V., Séries de Dirichlet, Gauthier-Villars, Paris, (1933).

[7] Ehrenpreis, L., Fourier Analysis in Several Complex Variables, Wiley Interscience, New York, 1970.

[8] Hörmander, L., Complex Analysis in Several Variables, North-Holland, American Elsevier, New York, 1973.

[9] Kashiwara, M., Kawai, T. and Kimura, T., Foundations of Algebraic Analysis, Princeton University Press, Princeton, 1986.

[10] Kawai, T., The Fabry-Ehrenpreis gap theorem and linear differential equations of infinite order, Amer. J. Math., 109 (1987), 57-64.

[11] - The Fabry-Ehrenpreis gap theorem for hyperfunctions, Proc. Jap. Acad. Sci., 60 (1984), 276-278.

[12] Leont'ev, A.F., Representation of functions by generalized Dirichlet series, Math. U.S.S. R. Izvestija, 6 (1972), 1265-1277.

[13] Levin, B. Ja., Zeros of Entire Functions, Translations of the A. M. S., Providence, 1980.

[14] Levinson, N., Gap and Density Theorems, A.M.S., New York, 1940.

[15] Squires, W. A., Geometric conditions for universal interpolation in $\hat{\mathcal{E}}^{\prime}$, Trans. Amer. Math. Soc., 280 (1983), 401-413.

[16] Struppa, D.C., The fundamental principle for systems of convolution equations, Memoirs A.M.S., 273 (1983).

[17] Struppa, D.C. and Meril, A., Convolutors in spaces of holomorphic functions, Complex Analysis II, Springer-Verlag Lecture Notes in Mathematics, 1276 (1987), 253-275.

[18] Taylor, B. A., Some locally convex spaces of entire functions, Proc. Symp. Pure Math., 11 (1968), 431-467. 